\title{
Humeral torsion and throwing proficiency in early human evolution
}

Susan G. Larson

Department of Anatomical Sciences, Stony Brook University School of Medicine, Stony Brook, NY 11794 8081

E-mail address: susan.larson@ stonybrook.edu

Keywords: Humeral retroversion; Shoulder range of motion; Overhead throwing; Homo erectus 


\section{Background}

In their recent paper, "Clavicle length, throwing performance and the reconstruction of the Homo erectus shoulder," Roach and Richmond (2015) test the hypothesis that relative clavicular length predicts scapular position and explore whether or not variation in relative clavicular length in a sample of modern humans from northwestern Kenya is correlated to throwing performance. Since the impetus to undertake these analyses is in part based on supposed claims I have made in my research on hominin shoulder evolution, I feel compelled to correct some misrepresentations they make of my work.

\section{The shoulder of Homo erectus}

The references I have made to relative clavicular length in my investigation of hominin shoulder evolution (Larson, 2007, 2009, 2013; Larson et al., 2007) concern a potential means of functionally interpreting the very low degree of humeral torsion typifying early hominins and early Homo. Contrary to the claim of Roach and Richmond (2015:2), I have not suggested that a more lateral position for the scapula in early Homo and an anterior facing shoulder joint was "necessary to allow early H. erectus to overcome deficits in internal shoulder rotation caused by very low humeral torsion." What I have argued is that the very low degree of humeral torsion that has been documented for all early hominins and early Homo would result in a reduced range of internal shoulder rotation if these taxa had a dorsal scapular position similar to that of modern humans. The difference here concerns what is cause and what is effect. I contend that given our current understanding of the factors influencing humeral torsion, the very low degree of torsion in early H. erectus is surprising, and to explain why I believe this to be the case I will digress briefly with the following overview.

\section{Humeral torsion}


Humeral torsion refers to the orientation of the proximal articular surface relative to the distal end of the humerus (Fig. 1). The use of the distal humerus as a reference is not simply a convenience. The elbow in virtually all mammals is a unidirectional hinge joint with little if any rotational mobility. What flexibility in hand positioning that exists in a taxon depends on the range of motion available at the shoulder coupled with forearm pronation/supination mobility. Therefore, the functional plane of elbow flexion/extension depends on the configuration of the glenohumeral joint. Intuitively, an elevated degree of humeral torsion (humeral head facing more inward) in humans and apes is related to the dorsal position of their scapulae since this results in more laterally facing glenoid fossae. The shift from a laterally positioned scapula on a dorsoventrally deep thorax typical of monkeys to a dorsal position on a broad thorax in hominoids is generally understood to be related to a greatly expanded range of motion at the shoulder due to the lateral reorientation of the shoulder joint. It is not actually known, however, whether selection for this increased range of motion caused the change in thoracic shape and scapular position, or if an enhanced range of motion was the fortuitous result of thoracic shape change bringing about scapular repositioning.

Modern humans and extant African apes are characterized by a high degree of humeral torsion compared to other primates (Fig. 2), and for many years this was counted as a shared derived feature of hominoids (e.g., Le Gros Clark, 1969; Andrews, 1985; Martin, 1986; Harrison, 1987). However, as Figure 2 shows, Asian apes have a much lower degree of torsion, though all apes have dorsally positioned scapulae. In 1988 I reported that the very low humeral torsion displayed by gibbons was likely related to the extreme degree of external shoulder rotation they exhibit during arm-swinging (Larson, 1988). The ability to achieve this degree of external rotation, however, incurred a certain cost in that it results in a "lateral-set" to their elbow joint that requires elevated recruitment of subscapularis as a medial shoulder rotator to overcome. In a subsequent study, I attempted to determine if the high level of torsion seen in modern humans and African apes was indeed a synapomorphy or was due to functional convergence by estimating the degree of humeral torsion displayed by the early hominin humeri known at the time, none of which were complete (Larson, 1996). My estimates of torsion for these incomplete humeri indicated much lower torsion than that of either modern humans or African apes, supporting an explanation of convergence for their similarity. In the years 
that have followed, several nearly complete hominin humeral specimens have been recovered (ARA-VP-1/4 and ARA-VP-7/2 [Ardipithecus ramidus; Lovejoy et al., 2009], MH1 and MH2 [Australopithecus sediba; Churchill et al., 2013], LB1/50 [H. floresiensis; Moorwood et al., 2005; Larson et al., 2007], and H. erectus KNM-WT15000 [Walker and Leakey, 1993; Larson et al., 2007], D2680, and D4507 [Lordkipanidze et al., 2007]), and these specimens confirm that very low humeral torsion characterized early hominins (Fig. 2).

The amount of humeral torsion displayed by an individual is a product of both development and function. Torsion increases from birth to maturity (Krahl, 1947; Edelson, 2000; Cowgill, 2007; see Fig. 3), and it is thought that variation in the degree of torsion between individuals may simply be a product of when this developmental trajectory ends (Yamamoto et al., 2006). Humeral torsion varies across different human groups, is often lower in males than in females, and is often lower in the dominant arm than the non-dominant arm (Martin, 1933; Krahl and Evans, 1945; Churchill, 1994; Edelson, 1999; see Fig. 4). Logically, the orientation of the humeral head and glenoid fossa must be related to some degree, but all modern humans share a dorsally placed scapula, although little is known about how differences in overall body form, shoulder breadth, chest size, or chest shape may subtly influence scapular position, which in turn might be correlated to variation in humeral torsion (a small amount of variation in glenoid orientation relative to the rest of the scapula — glenoid version — has been reported, but it does not appear to be correlated to humeral torsion; Matsumura et al., 2014). Nonetheless, "normal" glenohumeral configurations among humans apparently include a broad range of humeral torsion values. Functionally, this variation in humeral torsion has been associated with needed ranges of internal/external humeral rotation related to habitual activity patterns, with higher levels of torsion conferring an increase in the range in internal rotation, and lower torsion increasing the range of external rotation. Since the total range of rotational motion at the shoulder doesn't change, an increase in range of motion in one direction is bought at the expense of a loss in the other. Hence the "lateralset" to the gibbon shoulder due to their very low humeral torsion (Larson, 1988).

It was in the light of this developmental plasticity and functional adaptability that I found the unusually low degree of humeral torsion in fossil hominin taxa perplexing. In particular, it would have seemed likely that the shoulder of early $H$. erectus should display features related to manual manipulation of 
objects, reflecting a growing dependence on material culture for survival. With a dorsal scapula and laterally facing glenoid fossa, manipulation entails some amount of active internal shoulder rotation depending on the degree of humeral torsion. Since early Homo did not need the enhanced range of external humeral rotation for arm-swinging as gibbons do, it seems logical that the developmental plasticity of humeral torsion should result in them having at least a modest level of torsion.

The fact that all the nearly complete early hominin humeri uniformly display very low torsion (I have come to suspect that my estimates of torsion for proximal humeral fragments alone, i.e., A.L. 288-1r, OMO119-73-2718, and STS7, are too high) didn't make sense if they all possessed a human-like scapular position. While there is evidence that the earliest hominins maintained a shoulder positioned high above the thorax as in modern apes and presumably the last common ancestor (LCA), the scapulae of early H. erectus display more humanlike characteristics, suggesting they had moved to a lower position on the thorax. The simplest scenario would have been for the scapula to move inferiorly while maintaining its dorsal position, in which case the adaptability of humeral torsion should have resulted in an increase in torsion to accommodate a laterally facing glenoid fossa. The fact that early H. erectus nonetheless still displayed very low humeral torsion led to my hypothesis that in the course of this change in position, the pectoral girdle underwent a transitional phase in which the scapula moved not only inferiorly, but also somewhat anteriorly (Larson, 2007; Larson et al., 2007). Since the resulting more laterally positioned scapula did not appear to offer any particular advantage, I proposed that it might simply have been due to a clavicle that had not yet undergone the relative elongation observed in modern humans, by constraining scapular repositioning as it moved inferiorly on a flaring thoracic cavity. I suggested that the anterior shift in scapular position reported for people born with pathologically short clavicles (hypoplastic clavicle syndrome; Milgram, 1942; Guidera et al., 1991; Beals, 2000; Beals and Sauser, 2006) supported the plausibility of this scenario. As Roach and Richmond (2015) emphasize, this is indeed evidence that clavicular length can influence scapular position. However, to me, influencing scapular position does not necessarily imply being able to predict scapular position in humans, and I hope to now be able to correct this unintended extrapolation from my proposal. In fact I agree with Roach and Richmond (2015) that factors like thoracic shape and body build mitigate the 
likelihood of this being the case. In regard to the incidental complaint I noted by people with hypoplastic clavicles that they don't throw well (Guidera et al., 1991; Beals, 2000), their poor throwing proficiency has more to do with the restricted range of glenohumeral motion that results from their lateral scapular position and anteriorly oriented glenoid fossae than with clavicular length per se. Therefore, the investigation by Roach and Richmond (2015) demonstrating the lack of a relationship between clavicular length and throwing ability has little bearing on my analysis of hominin shoulder evolution. In any event, my proposal regarding a transitional phase in shoulder evolution does not claim that a more laterally positioned scapula was necessitated by low humeral torsion in early Homo as Roach and Richmond (2015) have reported; rather, I suggest that a more lateral position for the scapula was essentially a neutral by-product of other evolutionary changes, and low humeral torsion was an accommodation to the resulting more anteriorly facing glenoid fossae.

I concede, however, as Roach and Richmond (2015) have demonstrated, that relative clavicular length in early Homo is more variable than I expected, calling into question my proposal that the transitional shoulder configuration of a more laterally positioned scapula in early Homo was largely the result of a relatively short clavicle. This is not to say that this transitional phase did not occur, only that it may not have been brought about as a by-product of relative clavicular length. I maintain that without a strong selective force favoring the ability to put the shoulder into an extreme state of external rotation, with the plasticity and adaptability of humeral torsion, the need for internal humeral rotation with growing dependence on tools makes it highly unlikely that such low humeral torsion would be maintained throughout the first several million years of human evolution if all of these extinct hominin taxa had laterally facing glenoid fossae.

\section{Humeral torsion and overhead throwing}

In contrast to the perspective that very low humeral torsion in early $H$. erectus was an accommodation to a possibly selectively neutral anterior shift in scapular position, Roach and Richmond (2015; see also Roach et al., 2013) contend that scapular position in early H. erectus was like that of modern 
humans, and their very low degree of torsion was due to a very strong selective force favoring the ability to achieve extreme external rotation at the shoulder, namely, overhead throwing proficiency. The cocking phase of an overhead throw is achieved through extreme external shoulder rotation, and citing the well documented reduced humeral torsion in the throwing arms of modern day baseball pitchers, Roach et al. (2013) and Roach and Richmond (2015:6) contend that early H. erectus possessed a fully modern human-like shoulder that emphasized throwing proficiency as part of "the intensification of hominin carnivory and hunting behavior dating back 2 million years..." They argue that whatever minor cost this may have incurred in terms of the need for more active internal humeral rotation in other behaviors was more than offset by the survival value proficient throwing provided.

Figure 5 displays mean humeral torsion values from a number of studies of modern baseball players in general or pitches in particular illustrating the marked asymmetry in the degree of torsion observed in their dominant compared to their non-dominant arms, as well as the reduced torsion of their dominant arms compared to those of control samples. In addition to torsion, these studies typically also report corresponding asymmetries in the ranges of external shoulder rotation versus internal shoulder rotation. Also included in Figure 5 are average torsion values from Rhodes and Churchill (2009) on osteological samples of comparable modern human populations (only males have been included since the ball player subjects are all males). It is apparent that there is a great deal of variability in reported mean torsion values, and, unfortunately, much of this variation has as much to do with how torsion is measured as it does with differences between groups. To appreciate the impact of different approaches, it is necessary to again digress briefly to describe some of the differences in torsion measurement methods.

\section{Measuring humeral torsion}

Anthropologists and other morphologists have traditionally measured torsion on osteological specimens as the medial reorientation of the humeral head from a primitive condition of facing directly posteriorly, using the axis of the distal articular surface as a reference (e.g., Evans and Krahl, 1945; Krahl and 
Evans, 1945; Le Gros Clark and Thomas, 1951; Zapfe, 1960; Larson, 1996; see Fig. 1). Given that humeral torsion determines the functional axis for flexion/extension at the elbow, this seems a logical choice, but other reference axes have been used (see Fig. 1). In the human clinical and sports literature, however, more often than not humeral head orientation is being measured on living subjects and the metric most commonly employed is called humeral retroversion (or retrotorsion), which views a humeral head facing directly inward as the default condition, and increasing posterior deviation as increasing retroversion. Retroversion, therefore, is the inverse of torsion as used here: large retroversion angles correspond to small torsion angles, and vice versa (Fig. 1). In addition, studies documenting retroversion angles vary in which reference axis is used and also vary in the type of instrumentation used to visualize the humerus in living subjects. With CT imaging, axes of the proximal and distal ends of the humerus are identifiable in separate slices of the scan and are the most directly comparable to traditional torsion measurements on isolated humeri. Radiographic studies typically employ a single radiograph in semiaxial view showing both the humeral head and distal humerus, and use the transepicondylar line as a reference axis since the epicondyles are most visible. More recent studies documenting humeral retroversion use ultrasound technology to estimate humeral head orientation, but with an approach that differs in two major ways from traditional torsion measurements. First, the orientation of the humeral head is not measured directly; instead, a horizontal plane defined by the greater and lesser tubercles is used as a proxy (see Fig. 6). Second, although the distal transepicondylar line is the reference axis, it is also indirectly identified as a line assumed to be perpendicular to the axis of the subject's forearm (Fig. 6). Thus, humeral head orientation measured with ultrasound is not even directly comparable to other studies documenting retroversion in living subjects. Hernigou et al. (2002) compared several different methods of estimating humeral retroversion on cadaveric humeri and report that estimates using the actual transepicondylar axis are significantly smaller than those in which a line perpendicular to the forearm axis is used as a transepicondylar proxy. In other words, the ultrasound approach tends to over-estimate retroversion. The carrying angle of the elbow contributes to this discrepancy, tending to decrease with increasing humeral retroversion (Hernigou et al., 2002). 
In Figure 5, the reported retroversion angles have been converted to values for humeral torsion, which is the more commonly used metric in anthropology. To convert the ultrasound retroversion values I utilized the observation that the humeral head axis relative to a bisector of the bicipital sulcus forms an angle of about $120^{\circ}$ in most primates (dashed lines in Fig. 6; Larson, 1996), but this does not account for the influence of the elbow carrying angle, so these values likely under-estimate true torsion.

Placing the average humeral torsion values for baseball players displayed in Figure 5 in the context of these different measurement approaches, it is not surprising that the lowest values reported for the throwing arm of ball players come from ultrasound studies. Yet, even these probable under-estimates of humeral torsion are $15-20^{\circ}$ higher than those reported for early $H$. erectus. One-way ANOVA indicates that the mean for the three early $H$. erectus specimens is significantly different from that of the throwing arms of ball players (even including the values based on ultrasound) with $\mathrm{p}<0.001$. Nonetheless, Roach et al. (2013) emphasize that within the range of variation of modern day pitchers there are individuals with torsion values as low as those observed in early H. erectus, and Roach and Richmond (2015) argue that such low humeral torsion in throwing athletes is unproblematic since shoulder mobility allows them to overcome any correlated deficits in internal rotation.

The literature on injuries in throwing athletes, however, indicates that low humeral torsion is not unproblematic. The resulting loss of internal shoulder rotation range of motion is considered a contributor to shoulder injuries, and a metric known as glenohumeral internal rotation deficit (GIRD - typically defined as a $20^{\circ}$ or greater loss of internal rotation in the throwing arm compared to the non-throwing arm) has been identified as an injury risk predictor (Burkhart et al., 2003; Dines et al., 2009; Thomas et al., 2010; Whiteley et al., 2010; Myers et al., 2011; Wilk et al., 2011, 2012; Shanley et al., 2011; Kibler et al., 2012; Hibberd et al., 2014). Shanley et al. (2011) report that players with GIRD have a 4 to 5 times greater risk of upper limb injury. The types of injuries that have been associated with GIRD include ulnar lateral collateral insufficiency (Dines et al., 2009) as well as injuries to the rotator cuff and glenoid labrum (Burkhart et al., 2003), but perhaps most interesting in the current context is its association with scapular dyskinesis (scapular static and kinematic abnormalities), including increased protraction, anterior tilting, and decreased upward rotation 
(Burkhart et al., 2003; Borich et al., 2006; Thomas et al., 2010; Kibler et al., 2012). It appears, therefore, that in ball players with low humeral torsion there is a tendency for compensatory scapular repositioning that echoes the configuration I have suggested for the shoulder transitional phase in early H. erectus (Larson, 2007; Larson et al., 2007). For a pitcher, however, such changes can disrupt normal throwing mechanics and are associated with a variety of clinical problems (Kibler, 1998; Burkhart et al., 2003; Mihata et al., 2012). There are soft tissue factors that contribute to GIRD in addition to low humeral torsion, and as Polster et al. (2013) conclude, the interplay between the various factors is complex. In fact, they report a tendency for pitchers with less extreme retroversion (higher torsion) to experience more severe injuries, perhaps in their efforts to achieve the same degree of external shoulder rotation as those with lower humeral torsion. Polster et al. (2013:2019) suggest that humeral torsion of $135-150^{\circ}$ may represent a protective "sweet spot," and pitchers with torsion values outside this range are more prone to injury. It appears, therefore, that modern day overhead throwing athletes, perhaps particularly baseball players, are operating near the limits of shoulder configurations that combine an enhanced range of external shoulder rotation with some resistance to injury. As I believe Figure 5 demonstrates, this limit is approached with humeral torsion values well above those observed in early H. erectus.

It is impossible to know if modern day professional ball players throw more or less than members of an extinct hominin species that depended on hunting for survival. If indeed throwing proficiency was so essential for early $H$. erectus to warrant their very low humeral torsion values, why does humeral torsion increase among later hominins? A Middle Pleistocene humerus from Atapuerca (Carretero et al., 1997; Humerus II in Fig. 5) displays a level of torsion comparable to modern populations, as do Upper Paleolithic human samples (see Fig. 5). Indeed, the study by Rhodes and Churchill (2009) reporting these Upper Paleolithic torsion values as well as humeral torsion among Neanderthals was specifically undertaken to look for evidence of habitual use of throwing-based projectile weaponry by middle Upper Paleolithic times. They report that the level of bilateral asymmetry observed in the Upper Paleolithic samples is compatible with regular throwing but were hesitant to say this was conclusive evidence for habitual throwing due to the large amount of variance they observed. 


\section{Conclusions}

As illustrated in Figures 3, 4, and 5, in modern humans humeral torsion changes during development, and among adults varies between the right and left arms of an individual, between males and females of a single population, and in average values for different populations, but little is understood about the factors underlying this variation. Krahl (1947) suggested that the development of increasing humeral torsion during ontogeny reflects the influence of opposing muscular forces for internal and external rotation at the shoulder (see also Cowgill, 2007). Yet, comparisons of different modern human populations indicate that nonwestern populations with presumably higher activity levels often display lower average torsion values than western populations (Churchill, 1994; Larson, 2007). Our inclination is to functionally interpret differences in humeral torsion in terms of alterations in glenohumeral rotational ranges of motion, but torsion is also affected by the elbow carrying angle (Hernigou et al., 2002) and perhaps other subtle influences on scapular position.

The three known early $H$. erectus humeri are included in Figures 3-5 to demonstrate that, despite the considerable variability observed in modern humans, humeral torsion is markedly lower in these fossils. Unfortunately, the only behavior that has a documented functional connection to variation in humeral torsion is the overhead throwing motion, and, as the saying goes, when the only tool you have is a hammer, everything starts to look like a nail. There is little doubt that this unique ability has contributed to the success of the human lineage, but even if low humeral torsion is good (in this regard), it doesn't necessarily mean that even lower torsion is better. I contend that the distinctively low torsion observed in early H. erectus does not support the conclusion that the capacity for high speed throwing dates back nearly two million years.

\section{Acknowledgements}


I thank Bill Jungers for helpful comments on this manuscript. This work has been supported by the National Science Foundation Grant BCS 0935321.

\section{References}

Andrews, P., 1985. Family group systematics and evolution among catarrhine primates. In: Delson, E. (Ed.), Ancestors: The Hard Evidence. Alan R. Liss, New York, pp. 14-22.

Beals, R.K., 2000. The short clavicle syndrome. J. Pediatr. Orthop. 30, 389-391.

Beals, R.K., Sauser, D.D., 2006. Nontraumatic disorders of the clavicle. J. Am. Acad. Orthop. Surg. 14, 205214.

Borich, M.R., Bright, J.M., Lorello, D.J., Cieminski, C.J., Buisman, T., Ludewig, P.M., 2006. Scapular angular positioning at end range internal rotation in cases of glenohumeral internal rotation deficit. J. Orthop. Sports Phys. Ther. 36, 926-934.

Burkhart, S.S., Morgan, D.C., Kibler, B., 2003. The disabled throwing shoulder: spectrum of pathology Part III: the SICK scapula, scapular dyskinesis, the kinetic chain and rehabilitation. Arthroscopy 19, 641-661.

Carretero, J.M., Arsuaga, J.L., Lorenzo, C., 1997. Clavicles, scapulae and humeri from the Sima de los Huesos site (Sierra de Atapuerca, Spain). J. Hum. Evol. 33, 357-408.

Chant, C.B., Litchfield, R., Griffin, S., Thain, L.M.F., 2007. Humeral head retroversion in competitive baseball players and its relationship to glenohumeral rotation range of motion. J. Orthop. Sports Phys. Ther. 37, 514-520.

Churchill, S.E., 1994. Human upper body evolutionin the Eurasian later Pleistocene. Ph.D. Dissertation, University of New Mexico.

Churchill, S.E., Holliday, T.W., Carlson, K.J., Jashashvili, T., Macias, M.E., Mathews, S., Sparling, T.L., Schmid, P., de Ruiter, D.J., Berger, L.R., 2013. The upper limb of Australopithecus sediba. Science 340, 1233477-1-1233477-6. 
Cowgill, L.W., 2007. Humeral torsion revisited: a functional and ontogentic model for populational variation. Am. J. Phys. Anthropol. 134, 472-480.

Crockett, H.G., Gross, L.B., Wilk, K.E., Schwartz, M.L., Reed, J., O’Mara, J., Reilly, M.T., Dugas, J.R., Meister, K., Lyman, S., Andrews, J.R., 2002. Osseous adaptation and range of motion at the glenohumeral joint in professional baseball pitchers. Am. J. Sports Med. 30, 20-26.

Dines, J.S., Frank, J.B., Akerman, M., Yocum, A., 2009. Glenohumeral internal rotation deficits in baseball players with ulnar collateral ligament insufficiency. Am. J. Sports Med. 37, 566-570.

Edelson G., 1999. Variations in the retroversion of the humeral head. J. Shoulder Elbow Surg. 8, 142-145.

Edelson, G., 2000. The development of humeral head retroversion. J. Shoulder Elbow Surg. 9, 316-318.

Evans, F.G., Krahl, V.E., 1945. The torsion of the humerus: a phylogenetic study from fish to man. Am. J. Anat. 76, 303-337.

Guidera, K.J., Grogan, D.P., Pugh, L., Ogden, J.A., 1991. Hypoplastic clavicles and lateral scapular redirection. J. Pediatr. Orthop. 11, 523-526.

Harrison, T., 1987. The phylogenetic relationships of the early catarrhine primates: a review of the current evidence. J. Hum. Evol. 16, 41-80.

Hernigou, B., Duparc, F., Hernigou, A., 2002. Determining humeral retroversion with computed tomography. J. Bone Joint Surg. 84-A, 1753-1762.

Hibberd, E.E., Oyama, S., Myers, J.B., 2014. Increase in humeral retroversion accounts for age-related increase in glenohumeral internal rotation deficit in youth and adolescent baseball players. Am. J. Sports Med. 42, 851-858.

Kibler, W.B., 1998. The role of the scapula in athletic shoulder function. Am. J. Sports Med. 26, 325-337.

Kibler, W.B., Sciascia, A., Thomas, S.J., 2012. Glenohumeral internal rotation deficit: pathogenesis and response to acute throwing. Sports Med. Arthrosc. Rev. 20, 34-38.

Krahl, V.E., 1947. The torsion of the humerus: its localization, cause and duration in man. Am. J. Anat. 80, 275-319.

Krahl, V.E., Evans, F.G., 1945. Humeral torsion in man. Am. J. Phys. Anthropol. 3, 229-252. 
Larson, S.G., 1988. Subscapularis function in gibbons and chimpanzees: implications for interpretation of humeral head torsion in hominoids. Am. J. Phys. Anthropol. 76, 449-462.

Larson, S.G., 1996. Estimating humeral torsion on incomplete fossil anthropoid humeri. J. Hum. Evol. 31, $239-257$.

Larson, S.G., 2007. Evolutionary transformation of the hominin shoulder. Evol. Anthropol. 16, 172-187.

Larson, S.G., 2009. Evolution of the hominin shoulder: Early Homo. In: Grine, F.E., Fleagle, J.G., Leakey, R.E. (Eds.), The First Humans - Origins and Evolution of the Genus Homo. Vertebrate Paleobiology and Paleoanthropology Series. Springer, New York, pp. 65-75.

Larson, S.G., 2013. Shoulder morphology in early hominin evolution. In: Reed, K.E., Fleagle, J.G., Leakey, R.E. (Eds.), The Paleobiology of Australopithecus. Vertebrate Paleobiology and Paleoanthropology Series. Springer, New York, pp. 247-261.

Larson, S.G., Jungers, W.L., Morwood, M.J., Sutikna, T., Jatmiko, Saptomo, E.W., Due, R.A., Djubiantono, T., 2007. Homo floresiensis and the evolution of the hominin shoulder. J. Hum. Evol. 53, 718-731.

Le Gros Clark, W.E., 1969. The Antecedents of Man, $3^{\text {rd }}$ ed. Quadrangle Books, Chicago.

Le Gros Clark, W.E., Thomas, D.P., 1951. Associated jaws and limb bones of Limnopithecus macinnesi. Fossil Mammals of Africa, No. 3. British Museum of Natural History, London.

Lordkipanidze, D., Jashashvili, T., Vekua, A., Ponce de León, M.S., Zollikofer, P.E., Rightmire, G.P., Pontzer, H., Ferring R., Oms, O., Tappen, M., Bukhsianidze, M., Agusti, J., Kahlke, R., Kiladze, G., Martinez-Navarro, B., Mouskhelishvili, A., Nioradze, M., Rook, L., 2007. Postcranial evidence form early Homo from Dmanisi, Georgia. Nature 449, 305-310.

Lovejoy, C.O., Simpson, S.W., White, T.D., Asfaw, B., Suwa, B., 2009. Careful climbing in the Miocene: the forelimbs of Ardipithecus ramidus and humans are primitive. Science 326, 70-77.

Martin, C.P., 1933.The cause of torsion of the humerus and the notch of the anterior edge of the glenoid cavity of the scapula. J. Anat. 67, 573-582. 
Martin, L., 1986. Relationships among extant and extinct great apes and humans. In: Wood, B., Martin, L., Andrews, P. (Eds.), Major Topics in Primate and Human Evolution. Cambridge University Press, Cambridge, pp. 161-187.

Matsumura, N., Ogawa, K., Kobayashi, S., Oki, S., Watanabe, A., Ikegami, H., Toyama, Y., 2014. Morphologic features of humeral head and glenoid version in the normal glenohumeral joint. J. Shoulder Elbow Surg. 23, 1742-1730.

Mihata, T., Jun, B.J., Bui, C.N.H., Hwang, J., McGarry, M.H., Kinoshita, M., Lee, T.Q., 2012. Effect of scapular orientation on shoulder internal impingement in a cadaveric model of the cocking phase of throwing. J. Bone Joint Surg. 94-A, 1576-1583.

Milgram, J.E., 1942. Congenital forward shoulders (quadrupedal type) - treatment by clavicular osteotomy. Bull. Hosp. Joint Dis. 3, 93-96.

Morwood, M.J., Brown, P., Jatmiko, Sutikna, T., Wahuy Saptomo, E., Westaway, K.E., Awe Due, R., Roberts, R.G., Maeda, T., Wasisto, S., Djubiantono, T., 2005. Further evidence for small-bodied hominins from the Late Pleistocene of Flores, Indonesia. Nature 437, 1012-1017.

Myers, J.B., Oyama, S., Goerger, B.M., Rucinski, T.J., Blackburn, J.T., Creighton, R.A., 2009. Influence of humeral torsion on interpretation of posterior shoulder tightness measures in overhead athletes. Clin. J. Sport Med. 19, 366-371.

Myers, J.B., Oyama, S., Rucinski, T.J., Creighton, R.A., 2011. Humeral retrotorsion in collegiate baseball pitchers with throwing-related upper extremity injury history. Sports Health 3, 383-389.

Osbahr, D.C., Cannon, D.L., Peer, K.P., 2002. Retroversion of the humerus in the throwing shoulder of college baseball pitchers. Am. J. Sports Med. 30, 347-353.

Polster, J.M., Bullen, J., Obuchowski, N.A., Bryan, J.A., Soloff, L., Schickendantz, M.S., 2013. Relationship between humeral torsion and injury in professional baseball pitchers. Am. J. Sports Med. 41, 2015-2021.

Reagan, K.M., Meister, K., Horodyski, M.B., Werner, D.W., Carruthers, C., Wilk, K., 2002. Humeral retroversion and its relationship to glenohumeral rotation in the shoulder of college baseball players. Am. J. Sports Med. 30, 354-360. 
Rhodes, J.A., Churchill, S.E., 2009. Throwing in the Middle and Upper Paleolithic: inferences from an analysis of humeral retroversion. J. Hum. Evol. 56, 1-10.

Roach, N.T., Richmond, B.G., 2015. Clavicle length, throwing performance and the reconstruction of the Homo erectus shoulder. J. Hum. Evol. doi:10.1016/j.jhevol.2014.09.004 (in press).

Roach, N.T., Venkadesan, M., Rainbow, M.J., Lieberman, D.E., 2013. Elastic energy storage in the shoulder and the evolution of high-speed throwing in Homo. Nature 498, 483-487.

Shanley, E., Rauh, M.J., Michener, L.A., Ellenbecker, T.S., Garrison, J.C., Thigpen C.A., 2011. Shoulder range of motion measures as risk factors for shoulder and elbow injuries in high school softball and baseball players. Am. J. Sports Med. 39, 1997-2006.

Thomas, S.J., Swanik, K.A., Swaink, C.B., Kelly, J.D., 2010. Internal rotation deficits affect scapular positioning in baseball players. Clin. Orthop. Relat. Res. 468, 1551-1557.

Van Dongen, R., 1963. The shoulder girdle and humerus of the Australian aborigine. Am. J. Phys. Anthropol. $21,469-488$.

Walker, A., Leakey, R., 1993. The postcranial bones. In: Leakey, R., Walker, A. (Eds.), The Nariokotome Homo erectus Skeleton. Harvard University Press, Cambridge, pp. 95-160.

Whiteley, R.J., Ginn, K.A., Nickolson, L.L., Adams, R.D., 2006. Indirect ultrasound measurement of humeral torsion in adolescent baseball players and non-athletic adults: reliability and significance. J. Sci. Med. Sport 9, 310-318.

Whiteley, R.J., Adams, R.D., Nicholson, L.L., Ginn, K.A., 2010. Reduced humeral torsion predicts throwingrelated injury in adolescent baseballers. J. Sci. Med. Sport 13, 392-396.

Wilk, K.E., Macrina, L.C., Fleisig, G.S., Porterfield, R., Simpson, C.D., Harker, P., Paparesta, N., Andrews, J.R., 2011. Correlation of glenohumeral internal rotation deficit and total rotational motion to shoulder injuries in professional baseball pitchers. Am. J. Sports Med. 39, 329-335.

Wilk, K.E., Macrina, L.C., Arrigo, C., 2012. Passive range of motion characteristics in the overhead baseball pitcher and their implications for rehabilitation. Clin. Orthop. Relat. Res. 470, 1586-1594. 
Yamamoto, N., Itoi, E., Minagawa, H., Urayama, M., Saito, H., Seki, M., Iwase, T., Kashiwaguchi, S., Matsuura, T., 2006. Why is the humeral retroversion of throwing athletes greater in dominant shoulder than in nondominant shoulders? J. Shoulder Elbow Surg. 15, 571-575.

Zapfe, H., 1960. Die Primatenfunde aus der miozänen Spaltenfüllung von Neudorf an der March (Devinská Nová Ves). Tschechoslowakei. Schweiz. Palaeontol. Abhandlungen 78, 1-293. 


\section{Figure captions}

Figure 1. Humeral torsion measurements. A: axis of the humeral head; B: axis of the distal articular surface. In anthropology and other fields of morphological study, the primitive condition for humeral head orientation is assumed to be directly posterior (solid black line), and is expressed as either $0^{\circ}$ or $90^{\circ}$ relative to the articular surface (B). Increasing humeral torsion values reflect more inward orientations of the humeral head (dark grey angles). Some studies use a different distal reference axis, like the anterior surface of the distal articular surface (C; e.g., van Dongen, 1963; Edelson, 1999, 2000) or the transepicondylar axis (D; e.g., Osbahr et al., 2002; Reagan et al., 2002; Chant et al., 2007), that can affect the direct comparability of reported torsion values. In contrast, the human clinical and sports literature view a directly inward facing humeral head as the default condition and report posterior deviations from this orientation as increasing retroversion (light grey angles). Since retroversion is the inverse of torsion angles, increasing retroversion refers to decreasing humeral torsion and vice versa. To avoid confusion, all reported retroversion values from studies used here have been converted into torsion angles. Figure adapted from Larson et al. (2007).

Figure 2. Mean humeral torsion values ( \pm 1 standard deviation) in comparative samples of humans, apes, and monkeys, and in early hominin and early H. erectus fossils. For extant taxa, numbers in parentheses refer to sample sizes. Fossil and modern human data from: ${ }^{1}$ Lovejoy et al., 2009; ${ }^{2}$ Larson, $1996 ;{ }^{3}$ Churchill et al.,

2013; ${ }^{4}$ Larson et al., 2007 (error bars for hominin fossils represent error of the estimates); ${ }^{5}$ Lordkipanidze et al., 2007.

Figure 3. Ontogenetic changes in humeral torsion. The mean and confidence internal (CI) data for samples of juvenile humans are from Cowgill (2007). Numbers in parentheses refer to sample sizes.

Figure 4. Variation in mean humeral torsion values ( \pm 1 standard deviation) in modern humans. Data reported by Edelson (1999) are reproduced here to illustrate the variability in humeral torsion between the 
right (open symbols) and left (closed symbols) limbs of individuals, between males (black symbols) and females (grey symbols) of a single population, and between different populations. Numbers in parentheses refer to sample sizes. Torsion is typically lower for the right (presumably dominant) limb (although Krahl and Evans [1945] report the opposite), and in males compared to females (although not universally so). The metric reported by Edelson (1999) was retroversion using the anterior surface of the distal humerus as a reference axis (Fig. 1C), and those values have been converted to torsion angles here.

Figure 5. Mean humeral torsion values ( \pm 1 standard deviation) in the dominant and non-dominant arms of baseball players in comparable human osteological samples, plus humeral torsion for early $H$. erectus and a selection of later fossil hominins. Numbers in parentheses refer to sample sizes. Open symbols indicate the dominant arm or the right (presumably dominant) arm of the fossils or osteological samples. Grey symbols are for control samples included in a study of humeral torsion in ball players. All of the studies on ball players (and their control samples when included) as well as the comparative osteological samples reported humeral head orientation as retroversion angles, but these have all been converted to humeral torsion angles for ease of comparisons with data on hominin fossils. The studies of ball players have been grouped according to the type of instrumentation used to visualize the humerus in living subjects since this can influence the reported measurements. Fossil and modern human data from: ${ }^{1}$ Carretero et al., 1997; ${ }^{2}$ Rhodes and Churchill, 2009 (for fossil samples, only specimens for which both a left and right humerus from the same individual were available were included here); ${ }^{3}$ Whiteley et al., 2006; ${ }^{4}$ Myers et al., 2009; ${ }^{5}$ Myers et al., 2011; ${ }^{6}$ Osbahr et al., 2002; ${ }^{7}$ Reagan et al., 2002; ${ }^{8}$ Chant et al., 2007; ${ }^{9}$ Crockett et al., 2002.

Figure 6. Conventions used in measuring humeral retroversion using ultrasound. With the subject lying supine, the arm abducted $90^{\circ}$, and the ultrasound probe held vertically above the shoulder, the forearm is rotated until the greater and lesser tubercles are uppermost and lie in a horizontal plane (A). The axis of the humeral head is assumed to be perpendicular to this plane (D). The orientation of the forearm, usually determined with a goniometer placed along the subcutaneous surface of the ulna (B), is assumed to be 
perpendicular to a transepicondyle axis (C). Humeral retroversion is expressed as the angle $\alpha$ formed by the forearm axis relative to horizontal (A'). An individual with a humeral head facing directly posteriorly, that is, with a retroversion angle of $90^{\circ}$, should have a horizontal transepicondylar axis and a vertical forearm.

Decreasing values for $\alpha$ indicate decreasing retroversion angles (image on the right has a smaller retroversion angle than image on the left), or increasing values for humeral torsion. Although the noninvasive nature of ultrasound offers many advantages when studying living subjects, caution should be used when comparing ultrasound derived estimates of retroversion to ones based on other approaches due the different manner in which the proximal and distal humeral axes are defined. An additional confounding factor for ultrasound measurements of retroversion is the discrepancy between $\mathrm{C}$ and the actual transepicondylar axis due to the influence of the forearm carrying angle (Hernigou et al., 2002). 
Figure 1

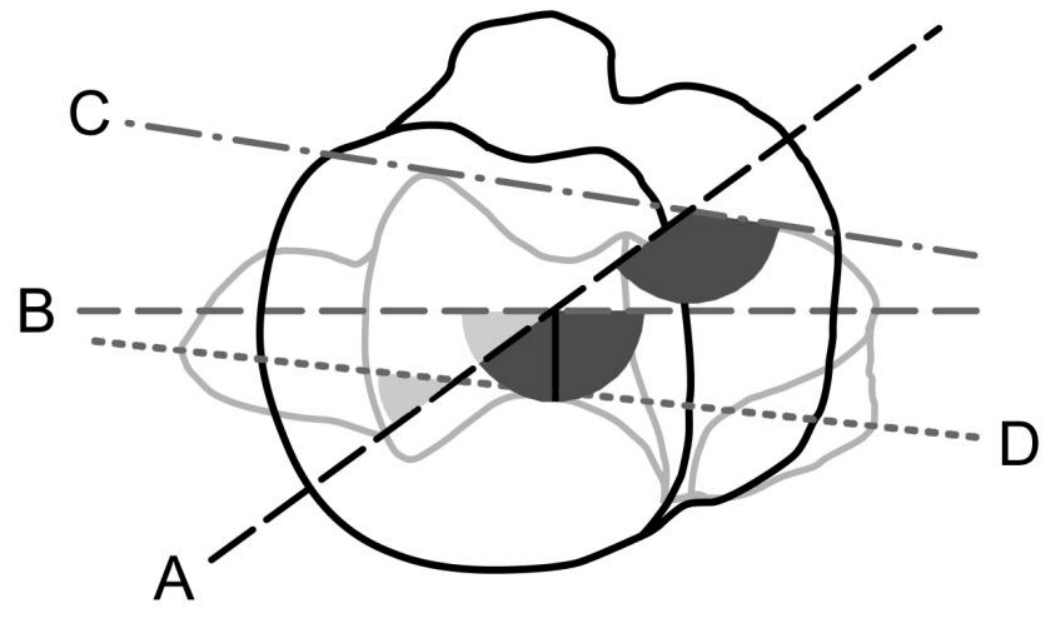


Figure 2

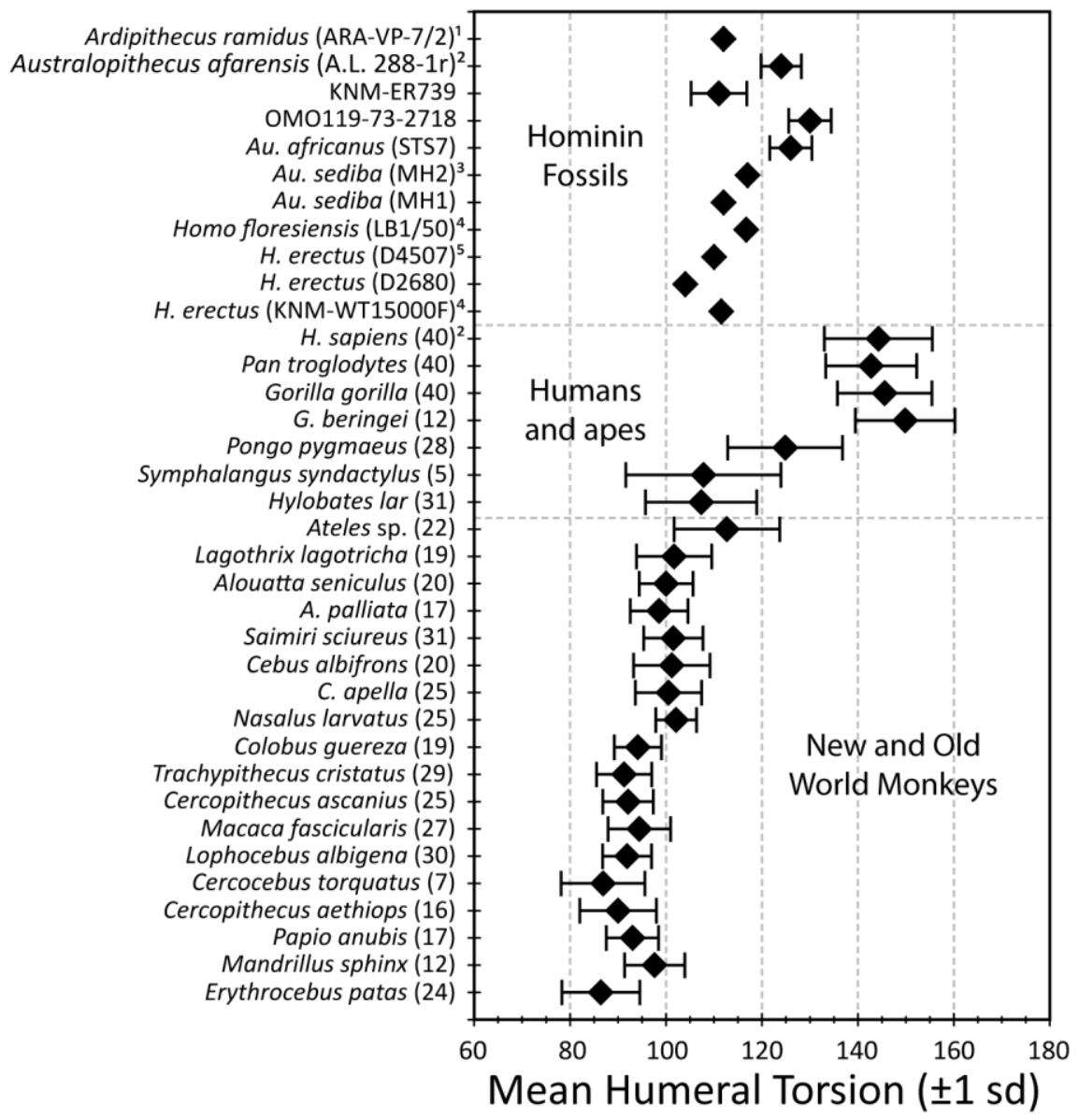


Figure 3

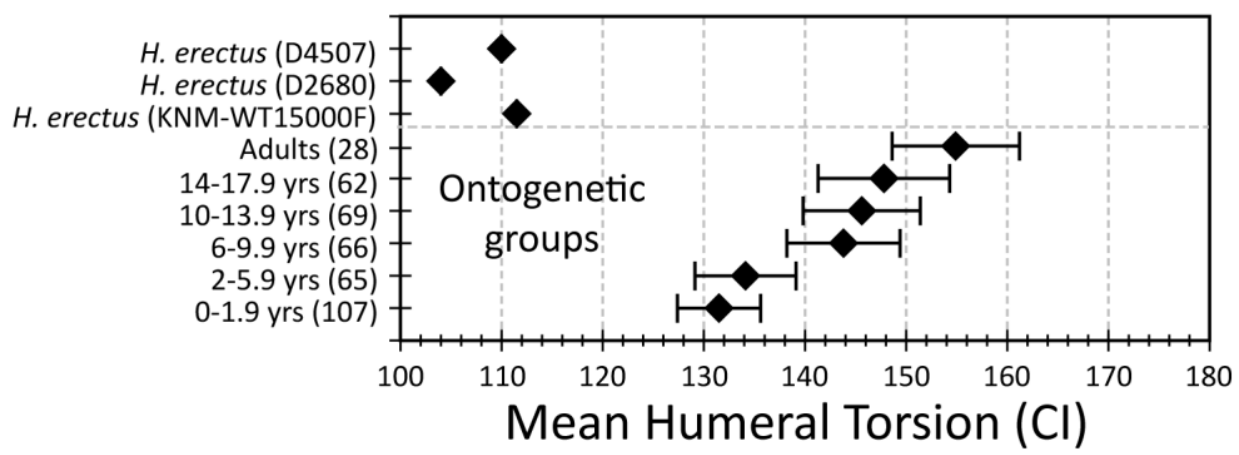


Figure 4

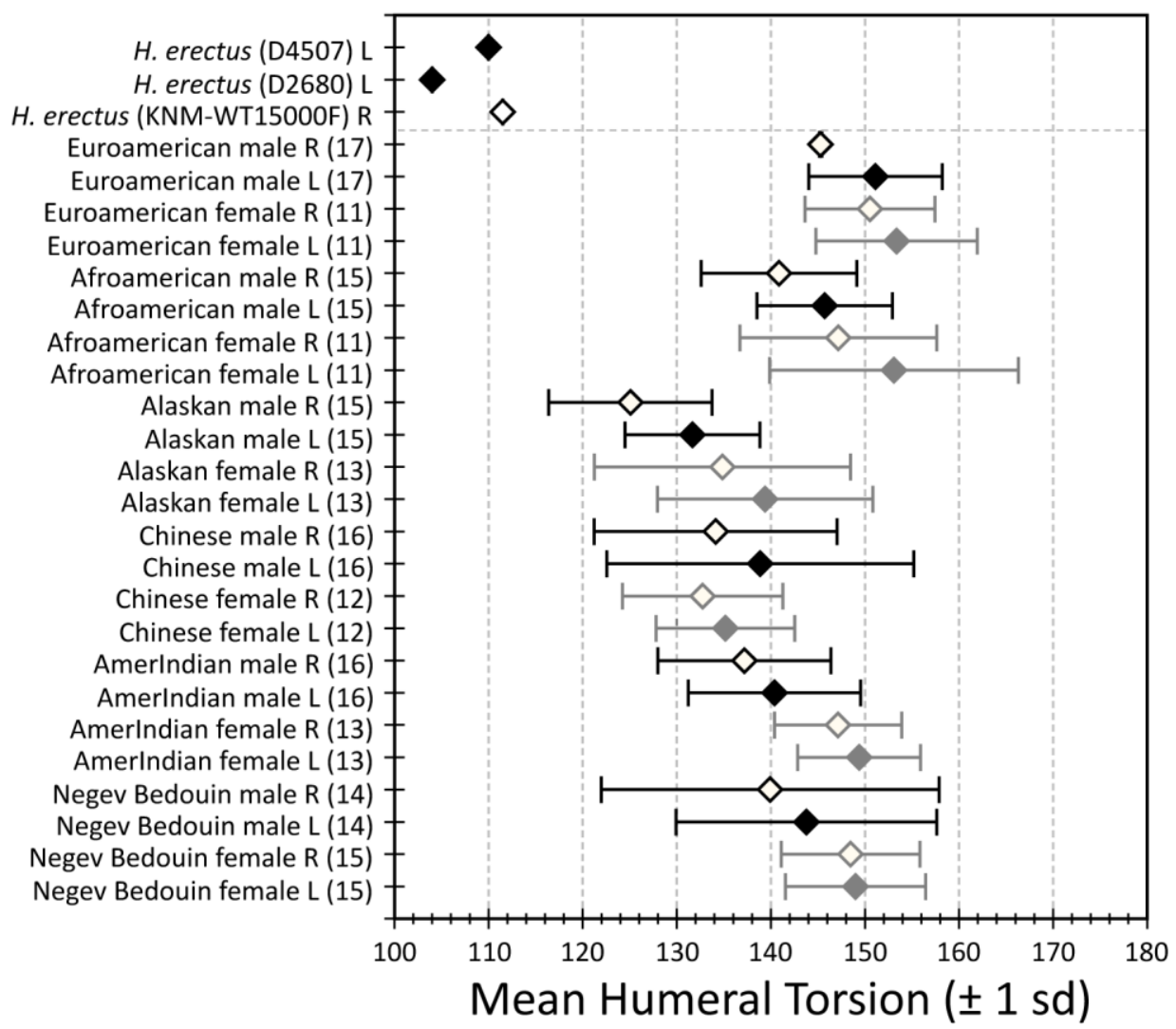


Figure 5

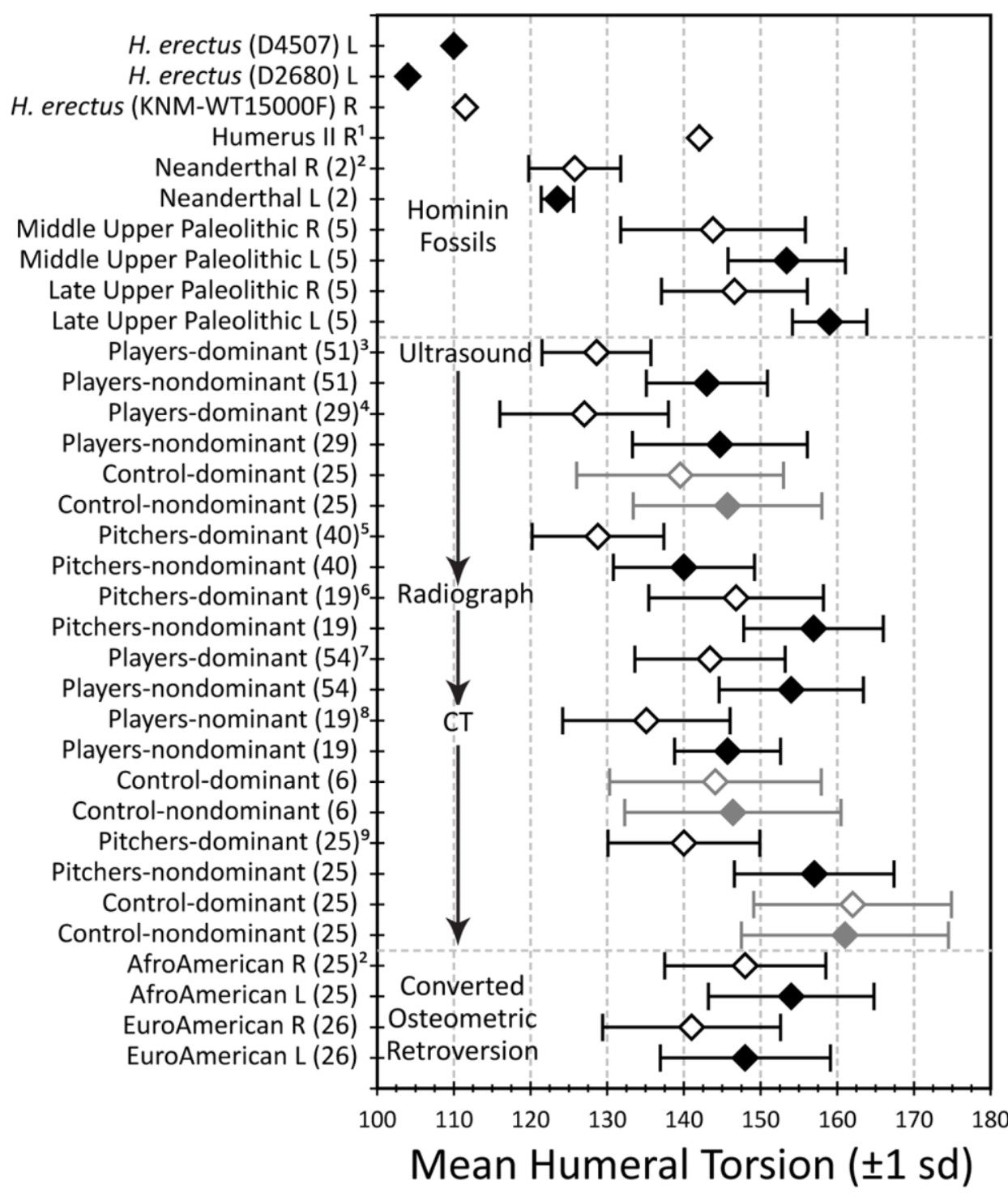


Figure 6
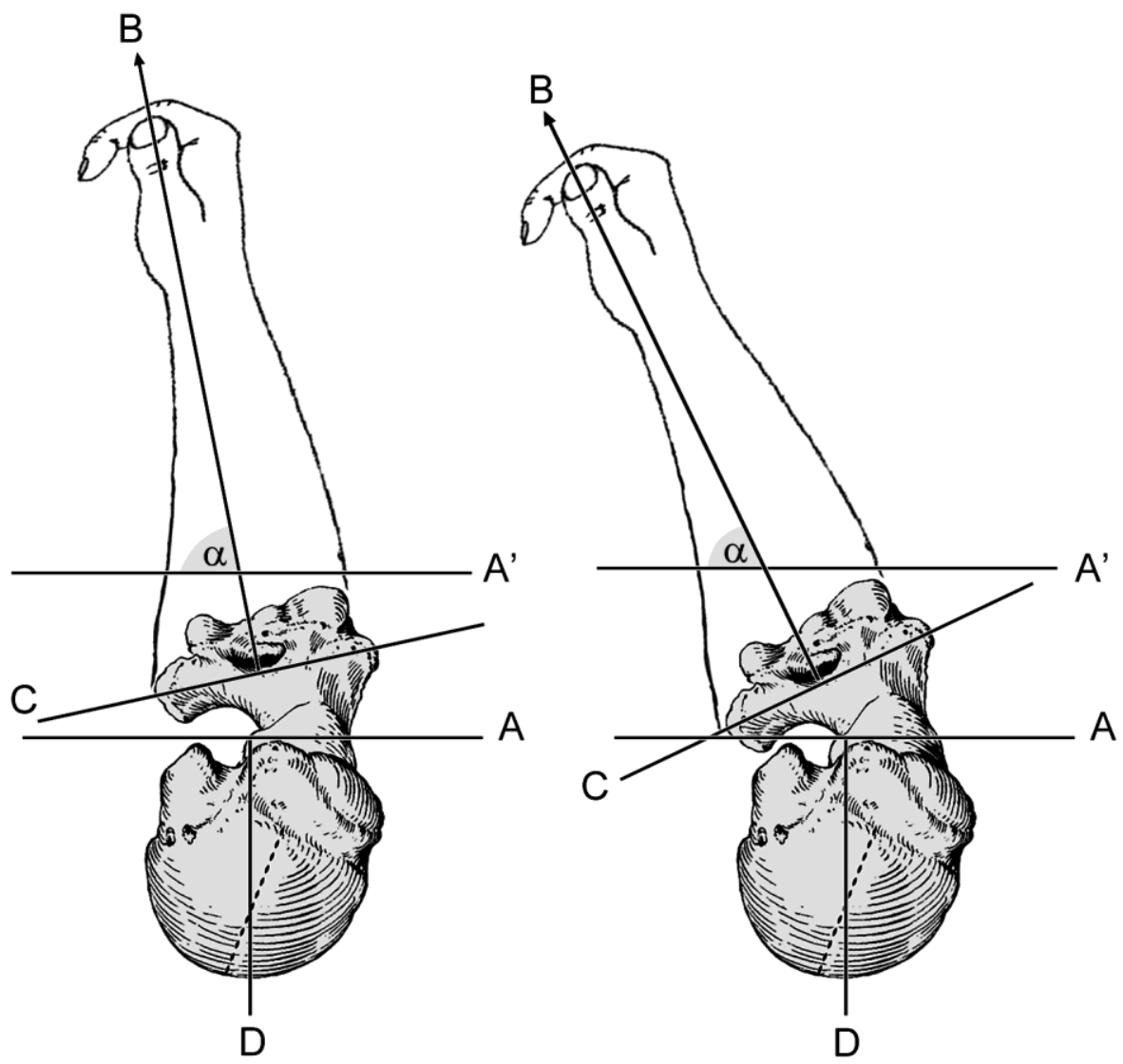